\title{
A Survey of No-Reference No-Training Based Image Quality Assessment Techniques
}

\author{
Piyush Joshi* \\ Indian institute of Technology Indore, India
}

Submission: May 29, 2017; Published: July 27, 2017

*Corresponding author: Piyush Joshi, Indian institute of Technology Indore, India Khandwa Road, Simrol, Madhya Pradesh 453552, India, Email: piyushjoshi3839@gmail.com

\begin{abstract}
Image quality can be defined as a measure to quantitatively estimate the perceptual quality of an image. The techniques available in the literature for image quality assessment (IQA) can be broadly divided into three categories: full reference (FR) based, reduced-reference (RR) based and no-reference (NR) based. In most of the practical applications, reference image of the distorted image is not available and hence NR based image quality estimation is the only possible way to estimate image quality. More significance of NR model lies in its resemblance with Human Visual System (HVS) as HVS also does not use any reference image for assessing the quality of an image. Mostly, NR models for quality assessment are based on training. However, training based techniques rely on training with provided human scores of distortions, and hence their scope is highly dependent on the distortions considered in the training. In literature, there are only few techniques which are training free. This paper surveys no-reference no-training based image quality assessment techniques. Results of all no-reference no-training IQA techniques are compared for noise and blur distortions.
\end{abstract}

\section{Survey}

A recent work on no-reference image quality assessment for multiple distortions is presented in [1] which does not use any training. This technique is based on the ability of the natural images to exhibit redundant information over various scales. A distorted image is considered as a deviation from the natural image. As the image is distorted more, the similarity of the original image with its down-scaled version will decrease more. Therefore, the dissimilarities of an image with its low-resolution versions are cumulated in the proposed method. This technique dissolves the query image into its scale-space and measure the global dissimilarity with the co-occurrence histograms of the original and its scaled images. These scaled images are the low pass versions of the original image. The dissimilarity, called low pass error, is calculated by comparing the low pass versions across scales with the original image. The high pass versions of the image in different scales are obtained by Wavelet decomposition and their dissimilarity from the original image is also calculated. This dissimilarity, called high pass error, is computed with the variance and gradient histograms and weighted by the contrast sensitivity function to make it perceptually effective. These two kinds of dissimilarities are combined together to derive the quality score of the query image.
A training free image quality assessment techniques are presented in SA-SS [2], SS-ZC [2]. This technique computes image quality based on structural activity information of different visual significance present in the image. This technique has assumed that human visual perception is highly sensitive to the structural information in a scene and has proposed the concept of structural activity (SA) together with a model of SA indicator in a new framework for no-reference (NR) image quality assessment $(\mathrm{QA})$ in this study The proposed framework estimates image quality based on the quantification of the SA information of different visual significance.

The technique named NOMDA [3] is motivated by two significant phenomena of perception in the retina of an eye. First being the center-surround retinal receptive field and second, existence of multiple spatial frequency channels. Centersurround retinal receptive field in the proposed technique is modeled with the help of Difference of Gaussians (DoG). In retina, multiple spatial frequencies have been found and due to this, signals generated from center and surround fields exist at different frequencies. In order to mimic center-surround receptive field at multiple frequencies, we compute multiple DoG images at multiple standard deviation values generated for 
different frequencies. Further, two significant features based on entropy and edges are extracted from the obtained DoG images and are subsequently used to compute the quality of the image.

\section{Results Comparison}

Experimental analysis of all techniques has been performed on Live [4] database. We consider images from all the databases which are distorted by Gaussian blur and white noise. The performance of all techniques has been evaluated based on realigned MOS/DMOS provided by the database. Evaluation metrics which have been used for analysis and comparison include root mean square error (RMSE) and Spearman RankOrder Correlation Coefficient (SROCC).

Table 1: Performance comparison with no-training based techniques on LIVE database.

\begin{tabular}{|c|c|c|c|c|}
\hline \multirow{2}{*}{$\begin{array}{c}\text { Quality } \\
\text { Measure }\end{array}$} & \multicolumn{2}{|c|}{ Gaussian Blur } & \multicolumn{2}{c|}{ White Noise } \\
\cline { 2 - 5 } & SROCC & RMSE & SROCC & RMSE \\
\hline SA-SS [2] & 0.888 & 7.876 & 0.959 & 6.839 \\
\hline SA-ZC [2] & 0.892 & 7.290 & 0.931 & 8.171 \\
\hline BIQES [1] & 0.9071 & 8.12 & 0.9694 & 7.713 \\
\hline NOMDA [3] & 0.9141 & 7.130 & 0.9786 & 5.650 \\
\hline
\end{tabular}

Table 1 presents comparison between all no-reference no-training based technique for white noise and Gaussian blur images respectively. The technique NOMDA [3] clearly outperforms all no-training based techniques such as SA-SS, SA$\mathrm{ZC}$ and BIQES for Gaussian blur and white noise distortions for both evaluation metrics viz. SROCC and RMSE Table 1.

\section{Conclusion}

There are many no-reference training based techniques are available in literature to estimate image quality. But, there are only few techniques exist which are no-reference and do not depend on training. This paper surveys no-reference no-training based image quality assessment techniques and compares their results for noise and blurs distortions. The conclusion of survey is that there is much research need to happen in the area of no-reference and no-training based image quality assessment techniques.

\section{References}

1. Saha A, Wu Q (2015) Utilizing image scales towards totally training free blind image quality assessment. IEEE Trans Image Process 24(6): 1879-1892.

2. Zhang J, Le T M, Ong S, Nguyen TQ (2011) No-reference image quality assessment using structural activity. Signal Processing 91(11): 25752588.

3. Joshi P, Prakash S (2017) Retina inspired no-reference image quality assessment for blur and noise. Multimedia Tools and Applications, pp. $1-20$.

4. http://live.ece.utexas.edu/research/quality

\section{Your next submission with Juniper Publishers} will reach you the below assets

- Quality Editorial service

- Swift Peer Review

- Reprints availability

- E-prints Service

- Manuscript Podcast for convenient understanding

- Global attainment for your research

- Manuscript accessibility in different formats

( Pdf, E-pub, Full Text, Audio)

- Unceasing customer service

Track the below URL for one-step submission https://juniperpublishers.com/online-submission.php 